\title{
Análisis de la temática sobre Educación Física escolar en el Posgrado en Educación stricto sensu en Brasil ${ }^{1}$
}

\section{An analysis of the topics on Physical Education in schools in graduate courses on Education stricto sensu in Brazil}

DOl: https://doi.org/10.32870/dse.v0i21.629

\author{
Ademir Faria Pires* \\ Verónica Gabriela Silva Piovani** \\ Jonas Spaciari Matioli*** \\ Juliana Pizani**** \\ leda Parra Barbosa-Rinaldi***** \\ Jorge Both*****
}

\begin{abstract}
Resumen
El objetivo del estudio es evaluar las disertaciones y tesis de los programas de Posgrado en Educación stricto sensu en Brasil, vinculadas al tema Educación Física escolar. La muestra fue de 171 trabajos (125 disertaciones y 46 tesis) elaborados entre los años 1980 y 2016. Para el análisis de los datos fue utilizada la estadística descriptiva y el test chi-cuadrado, con un nivel de significancia de $95 \%(\mathrm{p}<0.05)$. Se evidenció que la mayor producción de trabajos fue entre los años 2010 y 2016 (56.7\%) y que 69.0\% de los trabajos eran de Diagnóstico/Descripción, 19.9\% eran de Intervención y $11.1 \%$ de Fundamentación.
\end{abstract}

Palabras clave: Conocimiento - producción científica - educación y ciencia - educación básica - educación física.

\begin{abstract}
The study evaluated the academic productions focused in Scholar Physical Education from the Stricto Sensu Postgraduation in Education Programs of Brazil. Participated of the study 171 academic productions made between 1980 and 2016. For data analysis was used descriptive statistic and the chi-square test, that adopted a significant
\end{abstract}

1 En Brasil hay dos tipos de programas de posgrado: latu sensu, "en sentido amplio", cuando se trata de programas de especialización, y solo se obtiene un certificado al concluir el curso, avalado por la institución que lo impartió; strictu sensu, "en sentido limitado", que implica un programa con mayor rigor académico, sea ligado a la investigación, o bien, profesionalizante; se obtiene título (Maestro o Doctor) al finalizar el programa y está sometido a la aprobación del Ministerio de Educación. Se ofrecen fundamentalmente en universidades públicas (Nota de la traductora).

* Máster en Educación Física. Estudiante de doctorado del Programa de Posgraduación Asociado en Educación Física. Docente en el área de Gimnasia y Danza en la Universidade Paranaense (UNIPAR) - Unidade Cianorte. Brasil. afariapires@gmail.com

** Magíster en Educación Física. Estudiante de doctorado del Programa de Posgraduación Asociado en Educación Física. Docente Asistente del Centro de Ciências Humanas, Educación y Letras de la Universidade Estadual do Oeste do Paraná (UNIOESTE) - Brasil. veropiovani@hotmail.com

*** Licenciatura y Bachillerato en Educación Física. Universidade Estadual de Maringá (UEM). Brasil. jonasspaciarimatioli@gmail.com

**** Doctora en Educación Física. Universidade Federal de Santa Catarina (UFSC). Líneas de investigación: Escuela, Formación profesional/profesores, Currículum, Deporte Educacional y Prácticas corporales en la escuela. Brasil. jupizani@hotmail.com

***** Doctora en Educación Física. Universidade Estadual de Maringá (UEM). Líneas de investigación: Manifestaciones corporales (juego, danza y gimnasia) y Educación Física (dimensión educacional y formación profesional). Brasil. parrarinaldi@hotmail.com

****** Doctor en Educación Física. Universidade Estadual de Londrina (UEL). Líneas de investigación: Formación y Desarrollo profesional en Educación Física y Salud y calidad de vida de profesores. Brasil. jorgeboth@yahoo.com.br 
level of $95 \%(p<0,05)$. The results showed that the period between 2010 and 2016 had more works produced $(56,7 \%)$ and that $69,0 \%$ of the works were Diagnosis/Descriptive, $19,9 \%$ were of Intervention and $11,1 \%$ were of Grounding category.

Keywords: knowledge - scientific production - education and science - basic education - physical education.

\section{Introducción}

Al relacionar la producción del conocimiento con la existencia de los programas de posgrado en Educación Física, se puede afirmar que las disertaciones y tesis ${ }^{2}$ producidas en estos programas son el fruto más original, que demuestra la vida, organización e identidad de un campo de conocimiento y/o comunidad de investigación. Aún más, al volverse públicos, estos trabajos evidencian las discusiones, los conceptos fundamentales, procedimientos y técnicas considerados en la elaboración de nuevos saberes o versiones actualizadas en el conocimiento existente. Sin embargo, dentro del área de la Educación Física y Ciencias del Deporte existen críticas relacionadas con los productos, resultados y efectos de la producción de conocimiento en la práctica social. Entre estas críticas se encuentran: el carácter ensayístico con escasa base epistemológica; el predomino de los abordajes que enfatizan el objetivismo y fragmentación del conocimiento producido; la falta de referencias contextuales en la elaboración de problemas de investigación; y el bajo impacto en el campo de la acción pedagógica, entre otros (Molina Neto et al., 2006).

Además, conforme al estudio realizado por Manoel y Carvalho (2011), cuando se trata de los programas de posgrado en Educación Física en Brasil, se identifica que entre las líneas y proyectos de investigación registrados en el Informe de la Coordinación de Perfeccionamiento del Personal de Nivel Superior (CAPES) ${ }^{3}$ en el año 2009, el área biodinámica se destaca en comparación con la sociocultural y la pedagógica. Según los autores, tal hegemonía representa la valoración dada a las investigaciones pautadas en las ciencias naturales y la dificultad para problematizar la intervención, principalmente en el ámbito escolar.

Así, Bracht et al. (2011) apuntan que para contribuir directamente en el desarrollo del área de Educación Física es necesario el mapeamiento sobre el cual está siendo producida. Este tipo de trabajo permite identificar las posibles tendencias en las temáticas investigadas y los aportes teórico-metodológicos adoptados en los estudios.

La producción de conocimiento en el área de la Educación Física ha sido el núcleo de diversos estudios (Kokobun, 2003; Molina Neto et al., 2006; Bracht et al., 2012; Wiggers et al., 2015). Sin embargo, hay una carencia de estudios que presenten la asociación entre el campo de la

\footnotetext{
2 La academia brasileña acredita la Disertación como el trabajo final de titulación para obtener el grado de Maestro. La tesis es exigida para obtener el grado de Doctor (Nota de la traductora).

3 Por sus siglas en portugués: Coordenação de Aperfeiçoamento de Pessoal de Nível Superior - CAPES (Nota de la traductora).
}

Diálopos 
Educación Física - especialmente en el ámbito escolar- inserta en la discusión del área de la Educación. Esto, porque la mayoría de los estudios sobre producción de conocimiento en la Educación Física como tema demuestran una preocupación generalizada con lo se está generando en la propia área de la Educación Física.

Es posible señalar que el surgimiento de los primeros cursos de posgrado en Educación Física en Brasil impulsó la producción de conocimiento en esta área. Al mismo tiempo, el incremento de los programas de posgrado stricto sensu y la producción en el conocimiento en Educación Física fueron fruto de acciones del gobierno para formar maestros y doctores en el exterior, principalmente en Estados Unidos. No obstante, esta característica no fue exclusiva del área de la Educación Física, debido a la constitución de los posgrados en Brasil y a los patrones asumidos, influenciados por la formación de profesores e investigadores brasileños formados fuera del país (Santos y Azevedo, 2009).

Así, las publicaciones sobre Educación Física ocurrieron también en programas de maestría y doctorado en Educación, teniendo en cuenta que, según Rodacki (2017), fue a partir de la segunda mitad de la década de los setenta e inicios de los ochenta que comenzaron a surgir programas de posgrado específicos en el área de la Educación Física. Además de eso, a partir del incremento en el número de programas de Posgrado stricto sensu en Brasil, hubo un aumento significativo en la producción científica, mismo que generó inquietudes respecto a lo que se estaba produciendo en el área pedagógica de la Educación Física, aunque al mismo tiempo se reconocen las contribuciones de estos estudios.

A partir de lo anteriormente expuesto, para este estudio se optó por el análisis de las tesis y disertaciones existentes en Educación Física escolar, como insumos para propiciar la discusión sobre este campo en dicho contexto. Por lo tanto, el objetivo del trabajo fue analizar las tesis y disertaciones sobre la Educación Física escolar producidas en los programas de posgrado stricto-sensu en Educación en Brasil, con miras a la caracterización de la producción de conocimiento.

\section{Materiales y métodos}

Esta investigación se caracteriza como descriptiva (Gil, 2010) por tener como objetivo verificar de forma precisa la incidencia de aspectos relacionados con el área de Educación Física en las disertaciones y tesis de programas de posgrado en Educación en Brasil y establecer relaciones entre los fenómenos encontrados. El abordaje utilizado fue cuantitativo (Silva et al., 2011).

Con el objetivo de definir la muestra del estudio, en un primer momento se realizó el levantamiento de los datos para diagnosticar los programas de posgrado stricto sensu en Educación en el país, según el tipo de institución y su ubicación geográfica. Entre los 177 programas disponibles, fueron seleccionados los 25 mejor posicionados de acuerdo con su calificación en la Plataforma Sucupira, de la CAPES; entre ellos, 18 vinculados a las instituciones superiores de 
enseñanza pública y 7 en instituciones privadas. Se destaca que 14 programas eran de la región Sudeste, nueve de la región Sur, una en el Nordeste y una de la región Centro-Oeste (Brasil, 2017).

Las tesis y disertaciones evaluadas fueron realizadas en el periodo comprendido entre 1980 y 2016, aunque no todos los programas que formaron parte de la investigación estaban en funcionamiento desde la década de 1980. Por otra parte, la colecta de datos se realizó entre los meses de octubre de 2016 y enero de 2017.

Para el análisis del estudio, la muestra fue compuesta por 171 trabajos (125 disertaciones y 46 tesis), seleccionados en un primer momento a partir de la búsqueda online, utilizando el término "educación física escolar" en su título, resumen o palabras-clave. En un segundo momento, se utilizaron los siguientes criterios de inclusión: 1) que estuvieran disponibles de forma completa, con título, nombre del autor, año y resumen; 2 ) tener como foco de estudio la Educación Física escolar.

Se estableció una categorización de los trabajos teniendo como referencia la investigación realizada por Bracht et al. (2011); así, las producciones fueron denominadas y caracterizadas de la siguiente forma: 1) Fundamentación: relacionada con los aspectos fundacionales de la Educación Física escolar, en incluye estudios que problematizan las bases teóricas; 2) Intervención: comprende los trabajos que tratan sobre la acción pedagógica en su esencia, desde los elementos que anteceden la práctica hasta los estudios que abordan la práctica pedagógica concreta; 3) Diagnósticos/Descripciones: en esta categoría se incluyen las investigaciones que relatan lo cotidiano de la Educación Física escolar y sus peculiaridades; aquí se añaden las investigaciones empíricas y los relatos de experiencia.

Para el análisis de los datos se aplicó la estadística descriptiva (frecuencia absoluta y relativa) y la estadística inferencial. Para verificar las asociaciones entre las distintas variables en el estudio se utilizó la prueba Chi-cuadrada, adoptándose en todos los análisis el nivel de significación de 95\% $(p<0.05)$.

\section{Análisis y discusión de los resultados}

Los resultados evidenciaron que la mayoría de las investigaciones fue publicada entre 2010 y 2016 (56.7\%); vinculadas a instituciones públicas federales (46.2\%); producidas en la región Sudeste (60.8\%); disertaciones (73.1\%); clasificada como descriptivas/diagnóstico (69.0\%) (tabla 1). 
Tabla 1. Distribución de frecuencia de las variables en el estudio

\begin{tabular}{|c|c|c|}
\hline Variables & Categorías & $\mathrm{n}(\%)$ \\
\hline \multirow{2}{*}{ Periodo } & $1980-2009$ & $74(43 \cdot 3)$ \\
\hline & $2010-2016$ & $97(56.7)$ \\
\hline \multirow{3}{*}{ Tipo de Institución } & Privada & $36(21.1)$ \\
\hline & Pública Estatal & $56(32.7)$ \\
\hline & Pública Federal & $79(46.2)$ \\
\hline \multirow{3}{*}{ Región } & Sudeste & $104(60.8)$ \\
\hline & Sur & 49 (28.7) \\
\hline & Nordeste/Centro-Oeste & $18(10.5)$ \\
\hline \multirow{2}{*}{ Tipo de producción } & Tesis & $46(26.9)$ \\
\hline & Disertaciones & $125(73.1)$ \\
\hline \multirow{3}{*}{ Clasificación de los trabajos } & Intervención & $34(19.9)$ \\
\hline & Descripción/Diagnóstico & $118(69.0)$ \\
\hline & Fundamentación & $19(11.1)$ \\
\hline
\end{tabular}

Fuente: Elaborada por los autores.

El mayor volumen de producción de disertaciones, al compararse con la de tesis, se puede justificar por el propio proceso de formación que implica el posgrado, en el que los cursos de maestría comprenden un periodo de dos años, en contrapartida a los cuatro años en el doctorado, así como el número de vacantes ofrecidas para cada uno de los niveles formativos. Es importante mencionar que los programas se crearon inicialmente ofreciendo solo la maestría, para después ampliarlos hacia el doctorado.

Así también, se destaca que en los últimos seis años evaluados — periodo de 2010 a 2016 — se presentó el mayor número de trabajos. Entiéndase que el aumento de espacios de publicación de tesis y disertaciones en el área de Educación Física escolar, creados en los programas de posgrado en educación durante este periodo, puede estar relacionado con su incorporación al IV Plan Nacional de Posgrado (PNPG), ${ }^{4}$ pujante entre los años de 2005 y 2010, el cual tuvo como objetivo principal hacer avanzar el Sistema Nacional de Posgrado en el país, así como subsidiar la construcción e implementación de políticas públicas volcadas a la educación, ciencia y tecnología (Brasil, 2004; 2010).

Además de eso, otro aspecto que pudo haber influido en ese aumento es la entrada y/o migración de profesores del área de Educación Física para estos programas. En este sentido, Kunz (2012) afirma que algunos investigadores de las áreas sociocultural y pedagógica de la Educación Física, con reconocimiento nacional, migraron hacia otros programas de posgrado como los de Educación, debido al aumento de evaluación de las producciones científicas en el área.

4 Por sus siglas en portugués: Plano Nacional de Pós-graduação (Nota de la traductora). 
Vale destacar que en el ámbito de la Educación Física existe el debilitamiento del área sociocultural y pedagógica, especialmente en los programas de posgrado, relacionándose directamente con la manutención de la producción que es exigida. Esto, muchas veces es entendido como una adopción de criterios inadecuados para evaluar a los investigadores en las líneas pedagógica y sociocultural (Rosa y Leta, 2011). De esta forma, se debilita el área sociocultural y pedagógica, y fragmenta la Educación Física (Rigo et al., 2011).

También, Manoel y Carvalho (2011), en un estudio realizado a partir de los informes de programas de posgrado de la CAPES en el año 2009, señalan que, entre los proyectos de investigación registrados en el área de la Educación Física, la subárea pedagógica tenía aproximadamente $10 \%$ del total de proyectos. Y afirman que el proceso de exclusión gradual de los docentes de las subáreas pedagógica y sociocultural es fruto de un sistema de evaluación del posgrado que no considera la diversidad y la singularidad de la naturaleza de los objetos de investigación.

Al identificar el número de publicaciones en relación con el tipo de institución, se constató que las instituciones públicas federales, seguidas por las instituciones públicas estatales, fueron las más destacadas. En ese punto, es importante resaltar que la expansión del posgrado en Brasil estuvo ligada a la iniciativa pública federal, dado que en los años de 1970, por iniciativa del gobierno militar, fue instituido el Sistema Nacional de Posgrado (Santos y Azevedo, 2009). De hecho, las universidades públicas federales son impulsoras del avance del posgrado en educación, aunque recientemente se destaca el papel de las universidades públicas estatales en este proceso (Ramalho y Madeira, 2005).

El elevado número de producciones en las regiones Sudeste y Sur evidenció el desequilibrio geográfico en el origen de las investigaciones. Se destaca que la producción de conocimiento en educación en Brasil aumentó de forma importante en las últimas décadas, lo que está relacionado con la creación de programas de posgrado en educación y revistas científicas. Sin embargo, el aumento no es homogéneo en las distintas regiones del país ni en las diferentes instituciones de enseñanza superior y programas de posgrado en educación, concentrándose en la región Sudeste (Ramalho y Madeira, 2005; Meneghel et al., 2007; Santos y Azevedo, 2009). Además de eso, es preciso resaltar que los primeros programas de posgrado en educación fueron creados a partir de 1960, en instituciones federales y estatales en las ciudades de São Paulo y Río de Janeiro. Eso se explica por el hecho de ser ciudades que representan importantes centros económicos en el país, lo que habilita tener condiciones de promover el desarrollo en enseñanza e investigación a nivel posgrado (Meneghel et al., 2007).

En el área de la Educación Física también se observa la disparidad entre macrorregiones brasileiras, puesto que la producción de conocimiento en esa temática en Brasil está concentrada en las regiones Sudeste y Sur. Tal hecho limita el alcance de la producción, pues las particularidades económicas, sociales y culturales que afectan a las escuelas y la Educación Física son escasamente conocidas en las regiones Norte, Nordeste y Centro-Oeste (Wiggers et al., 2015).

Diólo@os 
Al evaluar los tipos de producción, se observó una elevada proporción de trabajos descriptivos. Se destaca que apenas $19.9 \%$ de las producciones estaban asociadas a la categoría Intervención y $11.1 \%$ con categoría Fundamentación (tabla 1).

En ese sentido, el estudio de Pizani (2016) que categorizó tesis y disertaciones con temática de Educación Física escolar producidas en Programas de Posgrado stricto sensu en Educación Física, encontró mayor producción de trabajos en la categoría Diagnósticos/Descripciones (70.4\%), seguidos de Intervención (25.7\%) y Fundamentación (3.9\%). Aunque la colecta de datos había sido realizada en programas de posgrado de áreas distintas, las investigaciones mantuvieron la característica de realizar diagnósticos de contextos, en contraste con las investigaciones de Intervención o Fundamentación.

Los estudios realizados con soporte empírico diferente, como los periódicos del área de Educación Física, evidencian algunos resultados que van al encuentro de los identificados en el presente estudio. En este caso, el trabajo de Wiggers et al. (2015) observó que la categoría Diagnósticos/Descripciones obtuvo 39.01\%, seguida por los de Intervención con 34.82\% y Fundamentación con $22.90 \%$.

Por otro lado, el trabajo elaborado por Bracht et al. (2011) constató que la mayoría de los trabajos publicados en nueve de las principales revistas del área de Educación Física en Brasil estaba dentro de la categoría de Fundamentación (45.6\%), seguida por la categoría Intervención (34.8\%), Diagnósticos/Descriptivos (17.3\%) y otros (2.3\%). Los autores atribuyen el predominio de la categoría Fundamentación al propio debate interno del campo académico de la Educación Física. Esto debido a que las revistas analizadas surgieron en un momento en el que la Educación Física pasaba por una crisis de identidad, y se buscaba que la Educación Física escolar fuera fundamentada a partir de cuestiones pedagógicas más allá de las didácticas.

En su momento, el estudio de Silva Júnior (2016) buscó artículos sobre las prácticas supervisadas en la licenciatura en Educación Física en periódicos nacionales, en el periodo desde 1996 hasta 2015; la categoría Intervención fue la que tuvo mayor incidencia (51.3\%), seguida por las categorías Diagnósticos/Descripciones (41\%) y Fundamentación (7.7\%). Es importante resaltar que la temática "prácticas supervisadas" está relacionada al aspecto de la práctica pedagógica y a la formación de profesores, lo que puede tener condicionada la mayor presencia de artículos en la categoría de intervención.

Antunes et al. (2005) realizaron un trabajo semejante al de Bracht et al. (2011), en periódicos nacionales e internacionales del área, utilizando una categorización diferente. Las categorías que establecieron son: Proceso Enseñanza-aprendizaje (45.2\%), Caracterización (26.5\%), seguidos de Finalidad (9.4\%), Formación de profesores (7.6\%) y Epistemología (4.0\%).

Betti et al. (2011), al utilizar la caracterización de Antunes et al. (2005) en periódicos nacionales, diagnosticaron que los estudios de Caracterización fueron los que más aparecieron, seguidos del Proceso Enseñanza-Aprendizaje, Formación de profesores, Finalidad y Epistemo- 
logía. De esta manera, se entiende que la preocupación de estas investigaciones está enfocada más en el diagnóstico del contexto de la Educación Física escolar que en las cuestiones de intervención, denotando el distanciamiento de la práctica escolar generado en las instituciones de enseñanza superior. Antunes et al. (2005) indagan acerca de esta cuestión, afirmando que hay un desajuste entre lo que es producido en las universidades y lo que realmente ocurre en el campo escolar.

Otro estudio realizado por Dias y Correia (2013), en el cual se evaluó la producción en revistas nacionales referente a la temática de la Educación Física en la enseñanza media durante el periodo de 2005 a 2010, se verificó que la mayoría de los trabajos tenían como foco el Proceso de Enseñanza-aprendizaje (25.8\%) y temáticas Indefinidas (25.8\%). Enseguida, se identificaron las categorías: Finalidad (22.6\%), Caracterización (19.35\%) y Epistemología (6.5\%).

De acuerdo con Manoel y Carvalho (2011), aún existe en el área de la Educación Física un debate acerca de la legitimidad académico-científica y el reconocimiento como práctica social. De esta manera, puede inferirse que en el caso del presente estudio, al situarse en el área de la educación, la preocupación con la legitimidad académico-científica no había sido tan recurrente y sí con aspectos relacionados a la práctica social (temáticas que se encuadran en la categoría Diagnósticos/Descriptivos e Intervención). Aún dentro de la propia área de la Educación Física brasileña, hubo cambios importantes en los últimos años, que han llevado a esfuerzos para articular las dimensiones de producción en el conocimiento e intervención. Para estos autores, la producción de conocimiento en el área no necesita dirigirse por la dicotomía teoría-práctica (Dias y Correia, 2013; Fensterseifer, 2015).

Para Wiggers et al. (2015), existe una tendencia inversa entre las categorías Fundamentación e Intervención; mientras que la primera se retrae, la segunda se desarrolla; aunque el trabajo de Bracht et al. (2011) es el único encontrado que presenta una constatación a la inversa. Para explicar este fenómeno, los autores manifestan que actualmente hay una búsqueda de equilibrio entre los enfoques de la Educación Física escolar, lo que puede ser una respuesta a la disminución de investigaciones en el ámbito de la didáctica que esta área vivió en décadas anteriores. Pero también puede significar el agotamiento del paradigma que ha nutrido a estos estudios, lo que demanda la introducción de nuevos abordajes teóricos (Betti et al., 2011).

Sin embargo, Silva Júnior (2016) alerta sobre el hecho de que investigaciones categorizadas como Diagnósticos/Descripciones en general, apenas describieron una situación, presentan limitaciones metodológicas y debilidades en la fundamentación teórica del análisis. De esta manera, no es posible superar discursos superficiales y comunes en el sistema educativo y de la disciplina de la Educación Física, consecuentemente. Sumado a esto, algunos autores afirman que cuando los estudios son de naturaleza descriptiva es posible que exista una utilización en el espacio escolar para atender intereses de investigación de los propios investigadores, sin que haya diálogo entre estos y los profesores (Antunes et al., 2005; Dias y Correia, 2013). 
Sin embargo, esta preocupación en el contexto de la Educación Física escolar denota el interes de los investigadores del área por la interferencia que las variables socioambientales tienen en el proceso de enseñanza-aprendizaje, lo que podría explicar la cantidad de estudios en esa categoría (Antunes et al., 2005). Diversos autores creen que sería necesario tener estudios pautados en el proceso de intervención y fundamentación, ya que las prácticas de enseñanza con escasa base epistemológica pueden ser limitadas y perjudicar los procesos de enseñanza-aprendizaje (Dias y Correia, 2013; Silva Júnior, 2016).

Al evaluar dichos trabajos conforme a las categorías de estudios, se constató una asociación significativa ( $p=0.025)$, ya que las investigaciones diagnósticas representan $72.8 \%$ de las disertaciones, comparado con $58.7 \%$ de las tesis. De hecho, el tiempo destinado a la realización de los cursos de maestría es menor, lo que induce a la realización de investigaciones diagnósticas, las cuales favorecen estudios de naturaleza aplicada y de menor duración. Es así que las tesis enfocadas a la fundamentación representan $21.7 \%$ y, en el caso de las disertaciones, solo $7.2 \%$ se clasifican en esta categoría (tabla 2).

Tabla 2. Tipos de estudios conforme tipos de trabajos, instituciones y periodo de publicación

\section{Tipos de estudios}

\begin{tabular}{|c|c|c|c|c|}
\hline \multirow[b]{2}{*}{ Variables } & \multicolumn{3}{|c|}{ Tipos de estudios } & \multirow[b]{2}{*}{$\mathrm{p}$} \\
\hline & $\begin{array}{c}\text { Intervención } \\
\mathrm{n}(\%)\end{array}$ & $\begin{array}{c}\text { Diagnóstico/Descripciones } \\
\mathrm{n}(\%)\end{array}$ & $\begin{array}{c}\text { Fundamentación } \\
\mathrm{n}(\%)\end{array}$ & \\
\hline $\begin{array}{l}\text { Tipos de } \\
\text { trabajos }\end{array}$ & & & & 0.025 \\
\hline Tesis & og (19.6) & $27(58.7)$ & $10(21.7)$ & \\
\hline Disertación & $25(20.0)$ & $91(72.8)$ & $09(7.2)$ & \\
\hline \multicolumn{2}{|c|}{ Tipo de institución } & & & 0.103 \\
\hline Privada & $03(8.3)$ & $28(77.8)$ & $05(13.9)$ & \\
\hline Estatal & $14(25.0)$ & $33(58.9)$ & og (16.1) & \\
\hline Federal & 34 (19.9) & $57(72.2)$ & $05(6.3)$ & \\
\hline Periodo & & & & 0.095 \\
\hline $\begin{array}{l}1980 \text { hasta } \\
2009\end{array}$ & $11(14.9)$ & $51(68.9)$ & $12(16.2)$ & \\
\hline $\begin{array}{l}2010 \text { hasta } \\
2016\end{array}$ & $23(23.7)$ & 67 (69.1) & $07(7.2)$ & \\
\hline
\end{tabular}

Fuente: Elaborada por los autores.

Se destaca que, al comparar el periodo de publicación de las producciones con las regiones brasileña, se identificó una diferencia significativa $(p=0.026)$, debido a que la mayoría de la pro- 
ducción científica de la región Sur se concentró entre los años de 2010 y 2016 (71.4\%), mientras que la mayoría de la producción de las regiones Nordeste y Centro-Oeste fue realizada hasta 2009 (61.1\%) (tabla 3).

Tabla 3. Periodos de la producción de trabajos conforme regiones geográficas, tipos de trabajos y categoría administrativa de las instituciones

\begin{tabular}{|c|c|c|c|}
\hline \multirow[b]{2}{*}{ Variables } & \multicolumn{2}{|c|}{ Periodo } & \multirow[b]{2}{*}{$\mathrm{p}$} \\
\hline & $\begin{array}{c}\text { Hasta } 2009 \\
\text { n (\%) }\end{array}$ & $\begin{array}{c}2010 \text { a } 2016 \\
\text { n (\%) }\end{array}$ & \\
\hline Regiones geográficas & & & 0.026 \\
\hline Sur & $14(28.6)$ & $35(71.4)$ & \\
\hline Sudeste & $49(47.1)$ & $55(52.9)$ & \\
\hline Nordeste/Centro-Oeste & $11(61.1)$ & $07(38.9)$ & \\
\hline Trabajos & & & 0.703 \\
\hline Tesis & $21(45.7)$ & $25(54 \cdot 3)$ & \\
\hline Disertación & $53(42.4)$ & $72(57.6)$ & \\
\hline \multicolumn{3}{|c|}{ Categoría Administrativa de la Institución } & 0.204 \\
\hline Privada & $12(33 \cdot 3)$ & $24(66.7)$ & \\
\hline Estatal & $29(51.8)$ & $27(48.2)$ & \\
\hline Federal & $33(41.8)$ & $46(58.2)$ & \\
\hline
\end{tabular}

Fuente: Elaborada por los autores.

El hecho de que los programas localizados en las regiones Centro-Oeste y Nordeste presentaran mayor cantidad de producciones hasta 2009, puede estar relacionado con la expansión del posgrado en educación que ocurrió a finales de la década de 1980 e inicio de los 1990 (Meneghel et al., 2007). Por otro lado, el aumento de la producción en la región Sur en el periodo de 2010 a 2016 puede estar asociado al hecho ya indicado, sobre cómo el posgrado en educación se volvió una opción para los estudios de investigadores del área de la Educación Física en el ámbito de las ciencias humanas (Manoel y Carvalho, 2007; Kunz, 2012).

\section{Consideraciones finales}

Al considerar los resultados de este estudio, se concluye que la producción de conocimiento en Educación Física escolar en los programas de posgrado stricto sensu presentó un crecimiento en los últimos años. Tal hecho puede ser relacionado a la migración y/o entrada de docentes de las áreas pedagógicas y socioculturales de la Educación Física a programas de posgrado, así como a la consolidación de los programas de posgrado stricto sensu en Educación en Brasil. 
En lo que se refiere al tipo de instituciones de enseñanza superior, se constató que el mayor porcentaje de producción se concentraba en las instituciones públicas, lo que señala la importancia que tienen las políticas públicas en la expansión dentro del sistema. Sin embargo, se observó una desigualdad entre regiones del país, donde la región Sudeste presenta mayor número de tesis y disertaciones publicadas frente a las demás regiones.

Esta disparidad entre regiones respecto a la producción académica, también puede ser fruto del desarrollo económico en cada región, puesto que hubo crecimiento elevado en el número de trabajos publicados en la región Sur de Brasil, mientras que en las regiones CentroOeste y Nordeste no se acompañó con el aumento en la producción de tesis y disertaciones sobre Educación Física escolar.

En relación con los tipos de estudios, se puede evaluar que el área de posgrado en educación presenta mayor número de publicaciones de trabajos con el interés de diagnosticar las cuestiones relacionadas a la cotidianidad escolar. Tal hecho puede relacionarse con la madurez de la propia área de la Educación Física escolar, que entiende que la producción del conocimiento no puede ser ajena de lo que sucede dentro de la escuela.

Finalmente, el estudio buscó contribuir con una reflexión sobre lo que se está generando en el área de la Educación Física escolar, y así, mejorar e impulsar nuevos estudios en el área que colaboren al conocimiento y práctica de la Educación Física escolar.

\section{Referencias}

Antunes, F. H.; L. E. Dantas; S. Bigotti; J. H. Tokuyochi; G. Tani; F. Brasil Kundrat; M. André (2005). Um retrato da pesquisa brasileira em Educação Física escolar: 1999-2003. Motriz, 11(3), 179184. http://www.rc.unesp.br/ib/efisica/motriz/11n3/11ELPa.pdf

Betti, M.; O. L. Ferraz; L. E. Dantas (2011). Educação Física Escolar: estado da arte e direções futuras. Rev Bras Educ Fís Esporte, (25), 105-115. http://dx.doi.org/10.1590/S1807$\underline{509201100050001}$

Bracht, V.; B. Faria; F. Almeida; F. Ghidetti; I. M. Gomes; M. C. Rocha; Th. Machado; U. Almeida; C. E. Moraes (2011). A Educação Física Escolar como tema da produção do conhecimento nos periódicos da área no Brasil (1980-2010): parte I. Movimento, 17(2), 11-34.https://doi. org/10.22456/1982-8918.19280

Bracht, V.; B. Faria; C. E. Moraes; E. Fernandes; F. Almeida; F. Ghidetti; I. M. Gomes; M. C. Rocha; Th. Machado; U. Almeida; V. M. Penha (2012). "A Educação Física Escolar como tema da produção do conhecimento nos periódicos da área no Brasil (1980-2010): parte Il. Movimento, 18(2), 11-37. https://doi.org/10.22456/1982-8918.30158 
Brasil (2004). Plano Nacional de Pós-Graduação (PNPG) 2005/2010. Brasília: Ministério da Educação, Coordenação de Aperfeiçoamento de Pessoal de Nível Superior. http://www.capes. gov.br/images/stories/download/editais/PNPG 2005 2010.pdf

Brasil (2010). Plano Nacional de Pós-Graduação (PNPG) 2011/2020. Brasília: Ministério da Educação, Coordenação de Aperfeiçoamento de Pessoal de Nível Superior. http://www.capes. gov.br/plano-nacional-de-pos-graduacao

Brasil (2017). Plataforma Sucupira. Brasil: Coordenação de Aperfeiçoamento de Pessoal de Nível Superior. https://sucupira.capes.gov.br/sucupira/public/consultas/coleta/programa/quantitativos/quantitativoles.jsf?areaAvaliacao $=38 \& a r e a C o n h e c i m e n t o=7080000$

Dias, D. I.; W. R. Correia (2013). A Educação Física no ensino médio como objeto de estudo da produção acadêmico-científica nos periódicos nacionais. Rev Bras Educ Fís Esporte, 27(2), 277-287. http://dx.doi.org/10.1590/S1807-55092013000200011

Fensterseifer, P. E. (2015). Produção do conhecimento em Educação Física: algumas reflexões a partir do Brasil. Educación Física y Ciencia, 17(2), 1-7.

Gil, A. C. (2010). Como elaborar projetos de pesquisa. São Paulo: Atlas.

Kokobun, E. (2003). Pós-Graduação em educação física no Brasil: indicadores objetivos dos desafios e das perspectivas. Rev Bras Ciênc Esporte, 24(2) 9-26. http://revista.cbce.org.br/index. php/RBCE/article/view/356/310

Kunz, E. (2012). Pós-graduação em Educação Física no Brasil: O fenômeno da hiperprodutividade e formação cultural". Kinesis, 30(1), 1-10. http://dx.doi.org/10.5902/010283085717

Manoel, E. J.; Y. M Carvalho (2011). Pós-graduação na educação física brasileira: A atração (fatal) para a biodinâmica. Educ. Pesqui, 37(2), 389-406. http://dx.doi.org/10.1590/S151797022011000200012

Meneghel, S. M.; F. Robl; J. Wassem (2007). Desafios da produção de conhecimento em educação perspectivas institucionais e de programas de pós-graduação. 30a Reunião Anual da Anped. Gtt 11-Política da Educação Superior. http://www.anped.org.br/sites/default/files/gt11-3572-int.pdf

Molina-Neto, V;; M. C. Günther Camargo; F. Bossle; E. Wittizorecki Schultz; R. M. Molina Kreusburg (2006). Reflexões sobre a produção do conhecimento em educação física e ciências do esporte. Rev Bras Ciênc Esporte, 28(1), 145-165. http://rbce.cbce.org.br/index.php/RBCE/article/viewFile/44/52

Pizani, J. (2016). Educação física e a educação integral e de tempo integral no Brasil. [Tese de Doutorado]. Maringá: Programa de Pós-graduação Associado em Educação Física Universidade Estadual de Londrina - Universidade Estadual de Maringá. http://nou-rau.uem.br/nou-rau/ document/?code $=\mathrm{vt} \mid \mathrm{s} 000224210$

Ramalho, B. L.;V.P.Madeira Carvalho (2005). A pós-graduação em educação no Nortee Nordeste: Desafios, avanços e perspectivas, Revista Brasileira de Educação, (30), 70-81. http://dx.doi.org/10.1590/ $\underline{\mathrm{S} 1413-24782005000300006}$

Diólo pos 
Rigo, L. C.; G. Ribeiro; P. Hallal (2011). Unidade na diversidade: Desafios para a Educação Física no século XXI. Rev Bras Ativ Fís Saúde, 16(4), 339-345. http://rbafs.org.br/RBAFS/article/ view/631

Rodacki, A. L. F. (2017). As Universidades Brasileiras e os 40 anos da Pós-graduação da EEFE-USP. Rev Bras Educ Fís Esporte, (31), 51-54. https://doi.org/10.11606/1807-55092017000nesp051

Rosa, S.; J. Leta (2011). Tendências atuais da pesquisa brasileira em educação física parte 2: A heterogeneidade epistemológica nos programas de pós-graduação. Rev Bras Educ Fís Esporte, 25(1), 7-18.http://dx.doi.org/10.1590/S1807-55092011000100002

Santos, A. L. F.; J. M. Azevedo Lins (2009). A pós-graduação no Brasil, a pesquisa em educação e os estudos sobre a política educacional: Os contornos da constituição de um campo acadêmico. Revista Brasileira de Educação, 14(42), 534-550. http://dx.doi.org/10.1590/S1413$\underline{24782009000300010}$

Silva Júnior, A. P. (2016). Configurações e relações estabelecidas no Estágio curricular supervisionado na formação inicial de professores de Educação Física. [Tese de Doutorado]. Maringá: Programa de Pós-graduação Associado em Educação Física Universidade Estadual de Londrina - Universidade Estadual de Maringá. http://nou-rau.uem.br/nou-rau/ document/?code $=\mathrm{vtl}$ S000225220

Silva, Sh. G.; G. Minatto; D. Fares; S. G. Santos (2011). Caracterização da pesquisa (Tipos de pesquisa). En Métodos e técnicas de pesquisa quantitativa aplicada à Educação Física, Florianópolis: Tribo da llha, 67-73.

Wiggers, I. D.; N. Reis Santana; L. Teixeira e Silva; L. Rodrigues; M. Mello Lima; T. Freitas da Costa; Th. Praça Rodrigues de Moura y Mayrhon; J. A. Farias (2015). Um raio-x da produção do conhecimento sobre educação física escolar: Análise de periódicos de 2006 a 2012. Movimento, 21(3), 831-845. https://doi.org/10.22456/1982-8918.50517 